\title{
Photodynamic Therapy: Role in Dentistry (A Brief Review)
}

\author{
Suharika Aavula ${ }^{1}$, Harveen Singh Kalra ${ }^{2}$ \\ ${ }^{1}$ Dr. M.G.R Educational and Research Institute, Chennai,Tamil Nadu, India. \\ ${ }^{2}$ Reader, Periodontology, Genesis Institute of Dental Sciences and Research, Ferozepur, Punjab, India. \\ *Corresponding Author: Harveen Singh Kalra, Reader, Periodontology, Genesis Institute of Dental Sciences and \\ Research, Ferozepur, Punjab, India.
}

\section{Abstract}

Bacteria form a biofilm that is the safest habitat for them. Pathogenic bacteria are the main etiological factor responsible for the failure of various treatments. Quorum sensing helps bacteria to communicate with each other and coordinates with their activity. Mechanical debridement with antimicrobial therapy is usually a conventional mode of periodontitis patients. Another effective tool is Photodynamic therapy for localized and superficial infections. This article summarizes the history, general principles, applications, indications, mechanism, and adverse effects of photodynamic therapy in various fields of dentistry.

Keywords: Biofilm, Antimicrobial therapy, Periodontitis, Photodynamic therapy.

\section{INTRODUCTION}

It has been known since the $20^{\text {th }}$ century that visible light has damaging action on microorganisms in the presence of the dye. More recently, Photodynamic Therapy has been introduced for its anti-microbial action. It is a non-invasive method that involves photosensitizer agents know as (photosensitizers) in thepresence of oxygen.Itisused in treatmentforvarious diseases such as in the patient with antibacterial, antifungal, antiviral drug-resistant, pocket debridement and gingivitis, chronic periodontitis, aggressive periodontitis, Oral cancer, peri-implantitis, in oropharyngeal candidiasis, disinfect of the root canal, mucosal infections, treating denture stomatitis, etc. It is also known as Photoactivated disinfection, Photoactivated chemotherapy. ${ }^{1}$

It involves three components:

1. Photosensitizers

2. Light

3. Oxygen

\section{Photosensitizers}

First-generation Photosensitizer was available in the 1970s. They may be ingested orally, applied topically, or injected intravenously depending upon the agents. They possess photo-physical, chemical, and biological characteristics. Their ideal characteristics are similar to the pure form of chemical composition. ${ }^{2}$ They have been classified based on their chemical structures are:

1. Tetrapyrroles: chlorophyll, porphyrins, phthalocyanines

2. Furocoumarins: xanthotoxin, bergaptene, psoralen, and its methoxyderivatives.

3. Tricyclic dyes: methylene blue, proflavine, acridine orange, erythrosine

They affect gram-positive bacteria by bearing a positive charge.

Some commercially available are Photofrin, Foscan, ALA.

\section{Light Sources}

Diode laser system is commonly used since it is easy to handle, cost-effective and portable. For the treatment of large areas, sources such as tungsten filament, xenon, quartz halogen are used which are non-coherent by nature. ${ }^{3}$ LEDs are being used because they are small, lightweight, and less expensive than typical light sources. They are mostly activated by red, blue light with a depth of penetration from $0.5 \mathrm{~cm}$ to atleast $1.5 \mathrm{~cm}$. 
Mostly Fiber optic catheters are used. Light source delivery depends upon its location and morphology of the affected site.

\section{History}

It was discovered accidentally at the beginning of the $20^{\text {th }}$ century. It was applied in the medical field for the inactivation of cells, micro-organism by lightactivation. The term photodynamic action was introduced in 1904 by Von Tappeiner, one of the photobiology. In 1911, Hausmann conducted a study on the biological effects of hematoporphyrin and reported on the effect of light and hematoporphyrin in red blood cells and describe skin reactions in mice that are exposed to light. In 1913, the same study was carried out on humans. Later Dougherlg and Marcus renamed it "Photodynamic therapy". ${ }^{4}$ PDT was first approved by the Food and Drug Administration in 1999 to treat pre-cancerous skin lesions of the face and scalp.

\section{PRINCIPLE}

Photodynamic therapy is based on the principle that the photosensitizer or the photoactive molecule binds with the target cell and can be activated with the light of a suitable wavelength. This process generates free radicals that act on the cell membrane of the microbial cell leading to its toxic effects. However, it has been less effective against gram-negative as compared to grampositive due to their special cell wall. ${ }^{7}$ The production of singlet oxygen is responsible for reacting with biological systems and destroying them. The energy for the excited state is about $220 \mathrm{kcal} / \mathrm{mol}$ above the ground state level.

\section{MECHANISM OF ACTION}

Before continuing the therapy, protective glasses should be worn by the patient, the assistant, and the operator.

1. Photosensitizer or its metabolic precursor is administered to the patient.

2. Most photosensitizers are activated by light between $630-700 \mathrm{~nm}$.

3. Photosensitizer gets activated by two mechanisms:

- Type-I: By transfer of hydrogen/electron directly from photosensitizer producing ions or by removal of electron forming free radicals. These free radicals react with oxygen forming highly reactive species.

- $\quad$ Type-II: It includes the use of triplet state photosensitizer which reacts with oxygen to form highly reactive oxygen known as singlet oxygen. This singlet oxygen interacts with bacteria to induce oxidative damage on the cell wall or cell membrane. ${ }^{5}$

- However, the Type-II method is the most accepted pathway for bacterial destruction as it is limited to a localized area without causing any destruction to adjust the area.

4. Upon irradiation with light of a specific wavelength, the photosensitizer undergoes a transition from a low energy ground state to the excited singlet state.

5. The photosensitizer may decay back to its ground state or may undergo a transition to a higher energy triplet state. ${ }^{6}$

6. This triplet state reacts with oxygen to generate singlet oxygen and free radicals leading to toxic effects on bacteria or tumor cells.

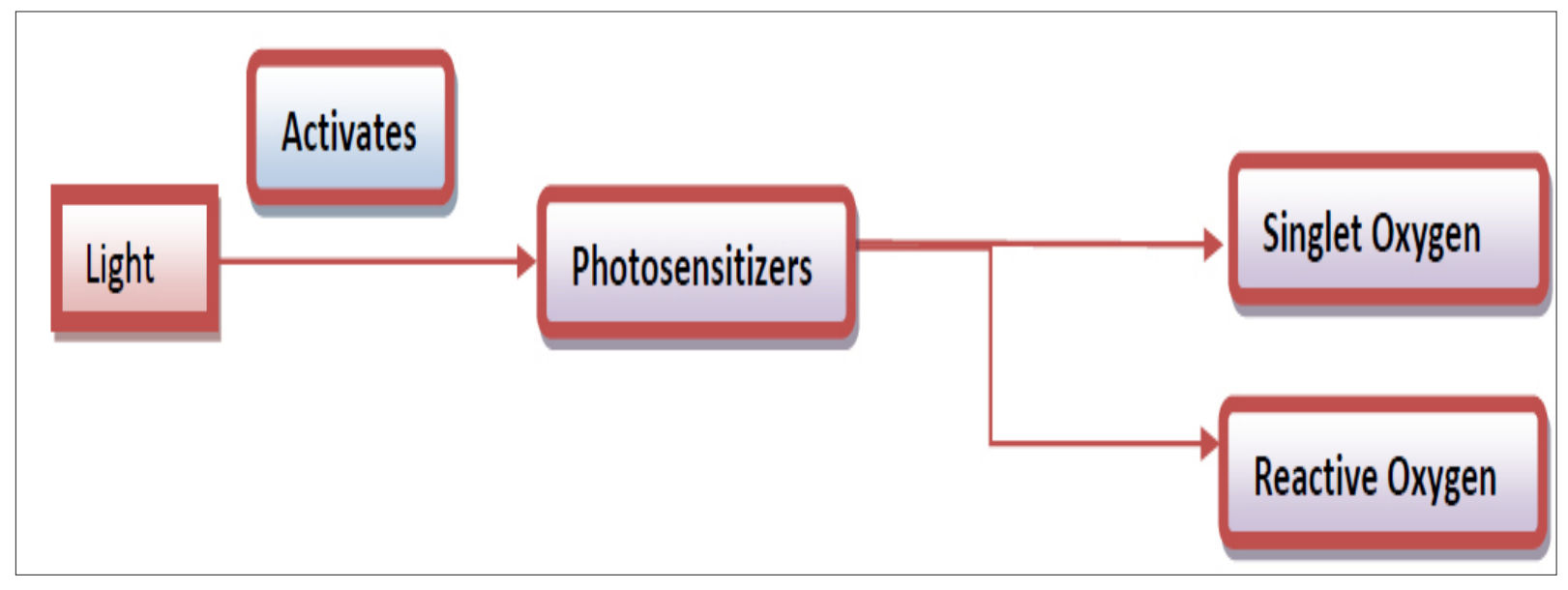




\section{Application in Photodynamic Therapy IN VARIOUS FIELDS OF DENTISTRY}

\section{Periodontology}

1. Scaling and root planing combined with photodynamic therapy using methylene blue leads to improvement of clinical trials.

2. They are useful in reducing redness, bleeding on probing and the number of $\mathrm{P}$. gingivalis bacteria.

3. In the treatment of Furcation area by decreasing the need for flap procedure and reducing treatment time.

4. It also detoxifies endotoxins such as lipopolysaccharide.

5. These lipopolysaccharide treated with this therapy does not stimulate the production of cytokines. Thus inactivates endotoxins. The photosensitizer compounds are topically applied in the gingival sulcus to destroy microbial pathogens. ${ }^{8}$

6. Peri-implantitis a localized inflammatory diseases affected bone and soft tissue affecting dental implant. Photodynamic Therapy helps in the treatment of decontamination to treat peri-implantits. ${ }^{9}$

\section{Oral Maxillofacial Surgery}

1. Useful in the diagnosis of pre-malignant and malignant lesions.

2. The lesion healed rapidly without any acute side-effects.

3. It has been successfully used in the treatment of infections like oral candidiasis, leukoplakia.

4. Use as an effective treatment of early stage tumors.

5. It is also used in the treatment and prevention of alveolar osteitis and post-extraction pain. ${ }^{10}$

6. It also helps in the treatment of herpes labialis. It also decreases the chances of the recurrence of vesicles.

7. Photodynamic Therapy along with laser treatment helps in surgical opening of implant site for cleaning and disinfection of local defects. ${ }^{11}$

\section{Endodontics}

Treatment of root canal includes three main steps access cavity, biomechanical preparation and obturation. Mechanical debridement of the canal helps to eliminate bacteria making the canal completely sterilized. However, reinfection occurs when complete elimination of bacteria is not there. This interferes with the healing of the tissue. Photodynamic Therapy along with chemo-mechanical debridement helps in eliminating chances for re-infection. It has shown promising results against endodontic bacteria. ${ }^{12}$ Treatment of cases with chronic periapical periodontitis with photodynamic therapy is also very successful.

\section{IN PHOTODYNAMIC ANTIMICROBIAL CHEMOTHERAPY}

Caries results due to an imbalance between demineralization and remineralisation. Bacteria from dental plaque releases acid which is responsible for demineralization. Photodynamic therapy thus helps in the removal of dental plaque as prevention for dental caries.

\section{Advantages}

1. Resistance to treatment does not develop with repeated treatment.

2. Has moderate side effects

3. Non -invasive technique

4. $\quad$ No need for anesthesia

5. Can target accurately or precisely in localized sites

6. Healing is without or little scarring

7. Improve the quality of life and lengthened survival

8. No damage to adjunct tissue

9. Shorter treatment tissue

10. Less or no need for flap raise

\section{Disadvantages}

Despite having so many advantages, it still has some disadvantages:

1. Cannot be used in patients with blood diseases such as porphyria.

2. Less effective in treating large tumors

3. Not effective against metastatic tumors

4. Pain relief medication is mandatory before or after the treatment. 


\section{Side EFFECTS}

Its side effects are divided into two categories related to the effects of light energy and the photochemical reaction.

1. Skin photosensitivity that can persist for weeks

2. Allergic reactions

3. Thermogenesis can occur if high-level lasers are used

4. Drugs make skin and eye sensitivity to light, the patient should avoid direct sunlight.

5. Photosensitizer can cause burns, swelling, scarring.

\section{FUTURE TRENDS}

Various in-vivo and in-vitro studies have been conducted and it has been found that photodynamic therapy is successful in various endodontic and periodontal problems. Peri-implantitis, mucosal infections, caries are their potential targets. However, Photodynamic therapy does not eliminate the need for chemotherapy but tends to accelerate the duration for treatment with low cost. Further studies are required to develop new photosensitizers, decrease the duration of photosensitivity, and effective light delivery systems so as decrease their side effects. Recent advances include a device based on LED technology allows for the production of light inside the target tissue. This technology expand the use of Photodynamic Therapy for the treatment of moderate and large volume of refractory tumors. ${ }^{16}$

\section{ConCLUSION}

Photodynamic therapy is a therapeutic approach with numerous advantages thus reducing longterm morbidity. Although the use of Photodynamic therapy in dentistry is very promising it is still in its infant stage. Superficial infection, bacterial and fungal infections seem to be an area that holds promise to incorporate this therapy as a treatment option. They are an excellent substitute for currently used chemotherapy. They can also be used as an alternative therapy for residual pocket treatment. ${ }^{17}$

\section{REFERENCES}

[1] Nagpal, S., Dodwad, V. and Vaish, S., 2012. Photodynamic therapy: a novel approach towards dentistry. J Pharmaceutical Biomed Sci (JPBMS), 21(21), pp.1-14.

[2] Pushpan SK, Venkatraman S, Anand VG, Sankar J, Parmeswaran D, Ganesan S, et al. Porphyrins in photodynamic therapy - A search for ideal photosensitizers. Curr Med Chem Anticancer Agents 2002;2:187-207.

[3] Kikuchi T, Mogi M, Okabe I, Okada K, Goto H, Sasaki $\mathrm{Y}$, et al. Adjunctive application of antimicrobial photodynamic therapy in nonsurgical periodontal treatment: A Review of literature. Int J Mol Sci 2015;16:24111-26.

[4] Dougherlg TJ, Marcus SL. Photodynamic therapy. Eur J Cancer 1992;28:1734-42.

[5] Surendranath P, Arjunkumar R. Low level laser therapy - A review. IOSR-J Dent Med Sci 2013;12:56-9.

[6] Sharman WM, Allen CM, van Lier JE (1999) Photodynamic therapeutics: basic principles and clinical applications. Drug Discov Today 4:507517.

[7] Nitzan Y, Shainberg B, Malik Z. Photodynamic effects of deuteroporphyrin on gram positive bacteria. J Microbiol 1987;15:251-8.

[8] Komerik N, Wilson M, Poole S. The effect of photodynamic action on two virulence factors of gram - negative bacteria. Photochem Photobiol 2000;72:676- 80 .

[9] Khammissa, R. a. G.; Feller, L.; Meyerov, R.; Lemmer, J. Peri-implant mucositis and periimplantitis: Bacterial infection. SADJ 2012, 67, $70,72-74$.

[10] Saini, R.; Lee, N.V.; Liu, K.Y.P.; Poh, C.F. Prospects in the Application of Photodynamic Therapy in Oral Cancer and Premalignant Lesions. Cancers (Basel) 2016, 8, 83.

[11] Neugebauer J (2005) Using photodynamic therapy to treat periimplantitis. Interview Dent Implantol Update 16:9-16.

[12] Demidova, T.N.; Hamblin, M.R. Effect of CellPhotosensitizer Binding and Cell Density on Microbial Photoinactivation. Antimicrob. Agents Chemother. 2005, 49, 2329-2335.

[13] Konopka K, Goslinski T (2007) Photodynamic therapy in dentistry. J Dent Res 86:694-707. 
[14] Vrouenraets MB, Visser GWM, Snow GB, van Dongen GAMS (2003) Basic principles, applications in oncology and improved selectivity of photodynamic therapy. Anticancer Res 23:505-522.

[15] Ormond AB, Freeman HS. Dye sensitizers for photodynamic therapy. Materials (Basel) 2013;6:817-40.
[16] Chen HM, Yu CH, Tu PC, Yeh CY, Tsai T, Chiang CP (2005b). Successful treatment of oral verrucous hyperplasia and oral leukoplakia with topical 5-aminolevulinic acid-mediated photodynamic therapy. Lasers Surg Med 37:114-122.

[17] Mielczarek-Badora E, Szulc M. Photodynamic therapy and its role in periodontitis treatment. Postepy Hig Med Dosw (Online) 2013;67:1058-65.

Citation: Suharika Aavula, Harveen Singh Kalra. Photodynamic Therapy: Role in Dentistry (A Brief Review). Archives of Dentistry and Oral Health. 2020; 3(2): 10-14.

Copyright: (C) 2020 Suharika Aavula, Harveen Singh Kalra. This is an open access article distributed under the Creative Commons Attribution License, which permits unrestricted use, distribution, and reproduction in any medium, provided the original work is properly cited. 